Krause, B., Looi, C. Y., Dresler, M., \& Cohen Kadosh, R. (in press). The neurochemistry of mathematical genius: reduced frontal excitation/inhibition balance in an expert calculator. Neuroscience

\title{
The neurochemistry of mathematical genius: reduced frontal excitation/inhibition balance in an expert calculator
}

Beatrix Krause ${ }^{\mathrm{a}}$, Chung Yen Looi ${ }^{\mathrm{a}}$, Martin Dresler ${ }^{\mathrm{b}}, \&$ Roi Cohen Kadosh ${ }^{\mathrm{a}}$

\author{
aDepartment of Experimental Psychology, University of Oxford, 15 South Parks Road, \\ OX1 3AQ, Oxford, UK \\ ${ }^{b}$ Donders Institute for Brain, Cognition and Behaviour, Radboud University \\ Nijmegen, 6525 HR Nijmegen, The Netherlands
}

Corresponding author:

Beatrix Krause

Late-life mood, stress, and wellness research program

Department of Psychiatry

Semel Institute for Neuroscience and Human Behavior

Geffen School of Medicine at UCLA

760 Westwood Plaza

Los Angeles, CA 90095

http://www.semel.ucla.edu/latelife

Email: BKrause@ucla.edu

Tel: +1 (310) 267-5119 


\begin{abstract}
Alterations in excitatory and inhibitory neurotransmitters (glutamate and GABA, respectively) have been found in various neuropsychiatric disorders, but have not been examined in individuals with prodigious cognitive abilities. Understanding exceptional brain processing is critical for developing biomedical interventions for cognitive and neurodevelopmental atypicalities. We tested the eleven-fold world champion in mental calculation, G.M., and compared his right middle frontal gyrus, which has been associated with mathematical prodigy, to four healthy control expert calculators, who were not prodigies. We found substantially lower frontal glutamate/GABA compared to non-prodigy controls, but not glutamate or GABA individually, measured with magnetic resonance spectroscopy. We suggest that prefrontal glutamate/GABA is a potential marker of extraordinary cognitive skills.
\end{abstract}




\section{Introduction}

A lightning calculator is typically an individual who, whenever presented with complex arithmetic problems (often multiplication, root calculation or prime factorization), provides the answer within only a few seconds. Hypotheses about the cause of such exceptional cognitive skills range from obsessive practice, to specific deficits giving rise to enhanced abilities in other domains (see (Heavey 2003), for a review). Specifically, one possible explanation is that prodigies lack higher-level cognitive abilities, which allows them to be highly efficient in certain simple, lower-level cognitive abilities (Snyder 2009). This theory has been supported by disrupting brain functioning in certain brain regions of normal participants and thereby improving performance on simple cognitive or perceptual tasks (Snyder, Mulcahy et al. 2003, Snyder, Bahramali et al. 2006). However, this theory is challenged by individuals with prodigious abilities who do not show impaired functioning in other cognitive domains and are therefore high-functioning.

Mental calculation is a highly complex concept involving a variety of brain functions, including a solid understanding of quantities, memory retrieval of arithmetic facts (ranging from multiplication tables to knowing that $\mathrm{p} i=\sim 3.14$, as opposed to calculating it), the manipulation of numbers in which intermediate calculation steps need to be held in memory and updated with concurrent results, and the comprehension and verbalization of number words. The brain network associated with such complex cognitive abilities is spread widely across the cortex, involving a variety of especially frontal and parietal regions (Arsalidou and Taylor 2011, Moeller, Willmes et al. 2015). Working memory capacity is strongly associated with calculation abilities (Furst and Hitch 2000, DeStefano and LeFevre 2004, Berg 2008) and can be linked to both the structure and activity of regions in the middle frontal gyrus (MFG). Specifically, the right MFG has been identified as one of the key regions in expert calculators (Pesenti, Zago et al. 2001, Fehr, Weber et al. 2010). Prodigious abilities have also been reported in high-functioning calculation experts (Pesenti, Seron et al. 1999, Fehr, Wallace et al. 2011). However, because much research on the cognitive and neural mechanisms associated with prodigious calculation skills has focused on savants with autism or other disorders, it is sometimes difficult 
to disentangle the characteristics of prodigious calculation as such from the characteristics of concomitant disorders. Therefore, the understanding of the underlying biological mechanisms associated with prodigious abilities is still limited.

Concentrations of GABA and glutamate can be measured non-invasively by magnetic resonance spectroscopy (MRS). Glutamate and GABA are the brain's major excitatory and inhibitory neurotransmitters, respectively, and are both strongly implicated in learning and plasticity (Trepel and Racine 2000, Ge and Dani 2005). In order for the brain to produce meaningful information processing and output, a balanced interaction between excitation and inhibition ( $E / I$ balance) is essential (Turrigiano and Nelson 2000). In humans, E/I balance as assessed by MRS has been associated with complex cognition, such as decision-making (Jocham, Hunt et al. 2012), face processing, and working memory (Cohen Kadosh, Krause et al. 2015). Superior cognitive functioning has been associated with higher frontal GABA and reduced glutamate in these studies, which suggests that lower frontal E/I is associated with superior cognitive abilities.

Here, we aimed to investigate glutamate/GABA in one of the rare highfunctioning lightning calculators, an individual with extraordinary cognitive capacity, Gert Mittring (G.M.) is the 11-fold world champion in mental calculation. We investigated glutamate and GABA levels in the right MFG, and in primary visual cortex (V1), as a control region. We compared G.M. with four male, age- and education-matched high-functioning expert controls without prodigious abilities. We expected that G.M.'s MFG, but not V1, would reveal reduced glutamate/GABA concentrations compared to controls. 


\section{Experimental procedures}

\section{Participants}

\section{Gert Mittring}

G.M. is a male 46-year old, high-functioning, healthy calculation and calendar calculation expert with no known history of neurological or psychiatric conditions. After more than 25 years of competing in international competitions and as a member of Mensa, G.M.'s holds eleven gold medals and several world records in mental calculation. He holds two university doctorate degrees in humanities, and a master's degree in computer science. His standardized mathematical abilities rank above the 99.8th percentile. Four healthy, righthanded age- and education-matched, male controls participated in the study (Table 1).

[Insert Table 1 here]

\section{Cognitive testing}

To assess basic mathematical abilities, we used the standardized Wechsler Individual Achievement Test (WIAT ${ }^{\circledR}$-II-UK, (Wechsler 2005)). In the subpart called 'numerical operations', increasingly difficult numerical operations are solved on the paper, while in subpart 'mathematical reasoning', the logical application of arithmetic to verbally and visually presented problems and scenarios is used. For each subtest, standard scores are identified according to the Wechsler age-standardized scoring tables. A composite score can be computed from the combined standard scores of the two subtests. While the subtests are correlated, they also assess different aspects of abilities; the numerical operations subtest requires good knowledge of a variety of different problem types, and the mathematical reasoning subtest requires more adaptive thinking. 


\section{MRS}

Single-voxel ${ }^{1} \mathrm{H}-\mathrm{MRS}$ was acquired (3T Verio Siemens, Erlangen, Germany) at the Oxford Centre for Functional MRI of the Brain. A 32-channel head coil was used. T1-weighted images were acquired (slice thickness $=1 \mathrm{~mm}, \mathrm{TR}=2040 \mathrm{~ms}$, $\mathrm{TE}=4.68 \mathrm{~ms}, \mathrm{TI}=900 \mathrm{~ms}$, flip angle: 8 degrees. The MRS sequence (spin-echo intensity acquired localized (SPECIAL, (Mekle, Mlynarik et al. 2009)), involved the acquisition of 128 averages with a TR=4000ms, a TE=8.5ms, a bandwidth of $2000 \mathrm{~Hz}$, and 4096 points (Mlynarik, Gambarota et al. 2006, Mekle, Mlynarik et al. 2009). Water suppression was performed using VAPOR (variable power radio frequency pulses with optimized relaxation delays) and 8 averages were acquired per voxel (Tkac and Gruetter 2005). Two 2x2x2cm voxels of interest were manually placed over the right MFG and V1 on axial and coronal structural slices. All voxels were placed by the same experimenter. The MFG was located between the inferior and superior gyri and anterior of the precentral gyrus on axial and coronal slice reconstructions (Figure 1). V1 was placed centrally across the interhemispheric fissure in the occipital lobe as posterior and inferior as the anatomy allowed the space between the cortex and the ventricles. Outer volume suppression (OVS) and measured $3 \times 3 \times 3 \mathrm{~cm}$. Acquisition time per voxel was approximately 15 minutes including sequence planning and shimming. Regional concentrations of GABA and glutamate concentrations raw and in relation to creatine are shown in Table 2. Cramer-Rao lower bounds (CRLBs) below $20 \%$ indicate the reliability of the acquired concentrations per region. Line widths (full-width-half-maximum, FWHM) and SNR are shown in $\mathrm{Hz}$ (Table 2). Due to the slight difference between voxels, no cross-voxel comparisons can be drawn.

\section{[Insert Figure 1 here]}

\section{Procedure}

Participants gave written informed consent prior to scan. Mathematical abilities were assessed in a separate session. Participants received $£ 10$ per hour for their time and effort. The study was approved by the Berkshire Research Ethics Committee. 


\section{Analysis}

\section{MRS}

In-house Matlab scripts were used for frequency drift correction, exclusion of bad averages beyond a standard deviation of 2.4 (see Table 2). We used the Linear Combination Model (LCM) (Provencher, 2001) version 6.2-2B, including eddy current correction and computations of the signal-to-noise ratio (SNR) and Cramer-Rao lower-bounds (CRLBs). Water suppressed and non-water suppressed spectra were included for the analysis. Corrections for gray and white matter and cerebrospinal fluid in the voxel were performed for GABA/creatine and glutamate/creatine. We used FMRIB's automated segmentation tool (FAST) to segment the T1-weighted images into 3D volumes of gray and white matter (GM and WM, respectively) and cerebrospinal fluid (CSF) (Zhang, Brady et al. 2001). The percentages of GM, WM and CSF were used to control for tissue volume within each voxel by multiplying the $\mathrm{GABA} / \mathrm{creatine}$ and glutamate/creatine ratios with $(\mathrm{GM}+\mathrm{WM}) /(\mathrm{GM}+\mathrm{WM}+\mathrm{CSF})$. We report these corrected values. Visual inspection of the voxels superimposed on the structural scans did not reveal reason for concern that voxels covered different anatomical regions in different participants.

We performed an additional region of interest (ROI) analysis to investigate whether G.M.'s anatomy differed from the one of the controls. We used Freesurfer version 6.0 to reconstruct cortical parcellations using the DesikanKillany atlas. The right caudal middle frontal gyrus served as the ROI. We performed a two-tailed Crawford's t-test on cortical volume. As an exploratory analysis, we also tested for cortical thickness, surface area and mean curvature in the ROI.

\section{Crawford's t-test}

Since we tested a single case and a small control group, the Crawford and Howell modified $t$-test was used to compare G.M.s regional GABA and 
glutamate concentrations with those of the four control participants (Crawford and Howell 1998). The degrees of freedom are calculated as:

$$
d f=N_{1}+N_{2}-2
$$

$\mathrm{N}_{1}$ represents the number of control participants, $\mathrm{N}_{2}$ in this case G.M., and accordingly, the degrees of freedom are equal to 3 . The $t$-statistic is then calculated by:

$$
t=\frac{\text { Glu }_{G M}-\overline{\text { Glu }_{\text {Controls }}}}{\text { Standard deviation }_{\text {Controls }} \sqrt{\frac{4+1}{4}}}
$$

Here, the mean of the control group is subtracted from G.M.'s glutamate concentration, and divided by the standard deviation of the control group multiplied by the root of the whole sample size divided by the control sample size. The software for the modified $t$-test is freely available and can be found online

(http://homepages.abdn.ac.uk/j.crawford/pages/dept/SingleCaseMethodsCom puterPrograms.HTM).

\section{Results}

Individual spectra are depicted in Figure 2. MFG Glutamate/GABA was significantly lower in G.M. than in the controls ( $(\mathrm{t}(3)=-3.39, p=.04)$ (Figure 3A). This effect was not due to GABA or glutamate alone (GABA: $t(3)=.2 .39, p=.1$; glutamate $\mathrm{t}(3)=.49, p=.66)$ and neither was creatine $(\mathrm{t}(3)=.56, p=.87)$. Additionally, there was no significant difference between CRLBs $(t(3)=-1.18$, $p=.32)$. There were no significant differences in tissue volume content between G.M. and controls within voxels of interest (see Table 2). Metabolite concentrations were corrected for tissue volume effects. There were no differences in V1 (Glutamate/GABA: $\mathrm{t}(3)=-.18, p=.87$; GABA: $\mathrm{t}(3)=.26, p=.81$, glutamate: $\mathrm{t}(3)=.48, p=.67$, Figure 3B). The SNR in V1 was significantly lower in G.M. compared to controls $(t(3)=-4.8, p=.02)$. Further differences in 
metabolite concentrations or measurement parameters were not found (see Table 2 for details).

\author{
[Insert Table 2 here] \\ [Insert Figure 2 here] \\ [Insert Figure 3 here]
}

The Freesurfer ROI analysis demonstrated that right MFG volume was not significantly different in G.M. compared to controls $(t(3)=1.41, p=.25$, also see Table 3). There were also no differences for mean curvature, cortical thickness and surface area $(\mathrm{t}(3)=.31, p=.78 ; \mathrm{t}(3)=.09, p=.93$ and $\mathrm{t}(3)=1.68, p=.19$, respectively). Lastly, in order control for potential differences in intracranial or whole-brain cortical volume, we further investigated those parameters and found no effects (total cortical volume: $\mathrm{t}(3)=1.46, p=.24$; intracranial volume: $\mathrm{t}(3)=.21, p=.85)$.

[Insert Table 3 here]

\title{
Discussion
}

We found reduced glutamate/GABA ratios in the right MFG of a single case of mathematical prodigy compared to non-prodigy controls. G.M.'s glutamate/GABA was $22.8 \%$ lower than the group average, and $17 \%$ lower than the lowest control. Furthermore, this finding was specific to the MFG, as it did not generalize to V1. Complementing previous functional and structural studies in calculation prodigies (Pesenti, Zago et al. 2001, Fehr, Weber et al. 2010), our results indicate that the right MFG of such highly skilled individual may also show a distinct neurochemical profile. In order to rule out that the difference in glutamate/GABA was merely related to differences in anatomy, i.e. cortical volume, we performed an ROI analysis. We found no evidence for differences 
in cortical morphology. It is therefore unlikely the current effect in glutamate/GABA ratios is driven by individual differences in structural anatomy. Furthermore, our results are also in line with previous studies in individuals with normal cognition, in which the association between glutamate and GABA has been considered (Jocham, Hunt et al. 2012, Michels, Martin et al. 2012, Cohen Kadosh, Krause et al. 2015). It has been suggested that MRS measures of E/I may reflect the processing efficiency of the area under investigation (Krause, Marquez-Ruiz et al. 2013). However, it is important to note that further advancement in the field of MRS is necessary to conclude that the quantification of GABA and glutamate in the current study reflects excitation and inhibition, as those measure do not purely reflect neurotransmitters. For instance, it has been discussed that the functional role of metabolite measurements with MRS is unclear (Rothman, Behar et al. 2003, Stagg, Bachtiar et al. 2011). Therefore, the observed concentrations are likely to consist of a combination of neurotransmitters as well as precursors that will be converted into other metabolic entities. It is therefore important to understand that the MRS measure of glutamate/GABA ratio does not necessarily reflect direct functional E/I balance, but instead more complex metabolic processes. Our finding suggests that glutamate/GABA assessed with non-invasive MRS may distinguish normal or skilled from exceptional calculators. Moreover, exploring the functional role of such neurochemical balances and their relationship with structural and functional neuroimaging would be valuable to get a better understanding of the neural mechanisms of prodigious abilities. While it is desirable to have a larger sample size of high-functioning prodigies to draw more substantial conclusions, the current scarcity of such individuals (Pesenti, Zago et al. 2001, Fehr, Weber et al. 2010) makes larger studies impractical.

Our current study demonstrates reduced right prefrontal glutamate/GABA ratios, in a high-functioning mental calculation prodigy compared to four control expert calculators. This effect was not found for individual concentrations of glutamate or GABA. To our knowledge, this is the first demonstration of altered brain neurochemical levels in superior cognition, in this case a high-functioning calculation prodigy. We suggest that extraordinary cognitive abilities may be 
reflected in MRS measures of glutamate/GABA in regions involved in complex calculation abilities. We hypothesize that the interplay between glutamate and GABA, rather than each metabolite separately, could provide information that could otherwise be missed. However, larger studies are essential in order to investigate this idea. Future research should explore the relationship between different biological substrates and mechanisms of high-level skills within and across cognitive domains for a better understanding of optimal brain and cognitive functioning, and whether modulating glutamate/GABA could lead to further improvement in cognitive abilities. 
Acknowledgements: We thank $\operatorname{Dr}$ Dr Gert Mittring for volunteering his time and effort for the study. This work was supported by the European Research Council (Learning\&Achievement 338065). BK was supported by the Economic and Social Research Council [grant number 1109458], the Deutscher Akademischer Austauschdienst (DAAD), and the Studienstiftung des Deutschen Volkes. 


\section{References}

Arsalidou, M. and M. J. Taylor (2011). "Is $2+2=4$ ? Meta-analyses of brain areas needed for numbers and calculations." Neurolmage 54(3): 2382-2393.

Berg, D. H. (2008). "Working memory and arithmetic calculation in children: the contributory roles of processing speed, short-term memory, and reading." Journal of experimental child psychology 99(4): 288-308.

Cohen Kadosh, K., et al. (2015). "Linking GABA and glutamate levels to cognitive skill acquisition during development." Human Brain Mapping: n/a-n/a.

Crawford, J. R. and D. C. Howell (1998). "Comparing an Individual's Test Score Against Norms Derived from Small Samples." The Clinical Neuropsychologist 12(4): 482-486.

DeStefano, D. and J. Ä. LeFevre (2004). "The role of working memory in mental arithmetic." European Journal of Cognitive Psychology 16(3): 353-386.

Fehr, T., et al. (2011). "The neural architecture of expert calendar calculation: a matter of strategy?" Neurocase 17(4): 360-371.

Fehr, T., et al. (2010). "Neural correlates in exceptional mental arithmetic-about the neural architecture of prodigious skills." Neuropsychologia 48(5): 1407-1416.

Field, A. (2009). Discovering Statistics using SPSS (and sex and drugs and rock ' $n$ ' roll). London, SAFE Publications Ltd.

Furst, A. J. and G. J. Hitch (2000). "Separate roles for executive and phonological components of working memory in mental arithmetic." Mem Cognit 28(5): 774-782. 
Ge, S. and J. A. Dani (2005). "Nicotinic Acetylcholine Receptors at Glutamate Synapses Facilitate Long-Term Depression or Potentiation." The Journal of Neuroscience 25(26): 6084-6091.

Heavey, L. (2003). Arithmetical savants. The Development of Arithmetical Concepts and Skills. A. Baroody and A. Dowker. N.J., Erlbaum: 409-434.

Jocham, G., et al. (2012). "A mechanism for value-guided choice based on the excitation-inhibition balance in prefrontal cortex." Nature neuroscience 15(7): 960-961.

Krause, B., et al. (2013). "The effect of transcranial direct current stimulation: a role for cortical excitation/inhibition balance?" Frontiers in human neuroscience 7(602).

Mekle, R., et al. (2009). "MR spectroscopy of the human brain with enhanced signal intensity at ultrashort echo times on a clinical platform at $3 \mathrm{~T}$ and $7 \mathrm{~T}$." Magn Reson Med 61(6): 1279-1285.

Michels, L., et al. (2012). "Frontal GABA levels change during working memory." PloS one 7(4): e31933.

Mlynarik, V., et al. (2006). "Localized short-echo-time proton MR spectroscopy with full signal-intensity acquisition." Magn Reson Med 56(5): 965-970.

Moeller, K., et al. (2015). "A review on functional and structural brain connectivity in numerical cognition." Frontiers in human neuroscience 9: 227.

Pesenti, M., et al. (1999). "Basic and Exceptional Calculation Abilities in a Calculating Prodigy: A Case Study." Mathematical Cognition 5(2): 97-148.

Pesenti, M., et al. (2001). "Mental calculation in a prodigy is sustained by right prefrontal and medial temporal areas." Nature neuroscience 4(1): 103-107. 
Rothman, D. L., et al. (2003). "In vivo NMR studies of the glutamate neurotransmitter flux and neuroenergetics: implications for brain function." Annu Rev Physiol 65: 401-427.

Snyder, A. (2009). "Explaining and inducing savant skills: privileged access to lower level, less-processed information." Philosophical Transactions of the Royal Society B: Biological Sciences 364(1522): 1399-1405.

Snyder, A., et al. (2006). "Savant-like numerosity skills revealed in normal people by magnetic pulses." Perception 35(6): 837-845.

Snyder, A. W., et al. (2003). "Savant-like skills exposed in normal people by suppressing the left fronto-temporal lobe." $\mathrm{J}$ Integr Neurosci 2(2): 149-158.

Stagg, C. J., et al. (2011). "What are we measuring with GABA magnetic resonance spectroscopy?" Communicative \& Integrative Biology 4(5): 573-575.

Tkac, I. and R. Gruetter (2005). "Methodology of H NMR Spectroscopy of the Human Brain at Very High Magnetic Fields." Appl Magn Reson 29(1): 139-157.

Trepel, C. and R. J. Racine (2000). "GABAergic modulation of neocortical longterm potentiation in the freely moving rat." Synapse 35(2): 120-128.

Turrigiano, G. G. and S. B. Nelson (2000). "Hebb and homeostasis in neuronal plasticity." Current opinion in neurobiology 10(3): 358-364.

Wechsler, D. (2005). Wechsler Individual Achievement Test (WIAT-II), Pearson Assessment.

Zhang, Y., et al. (2001). "Segmentation of brain MR images through a hidden Markov random field model and the expectation-maximization algorithm." IEEE Transactions on Medical Imaging 20(1): 45-57. 


\section{Figure 1}

Voxel position in the right middle frontal gyrus (MFG) on coronal (left) and axial (right) slices.

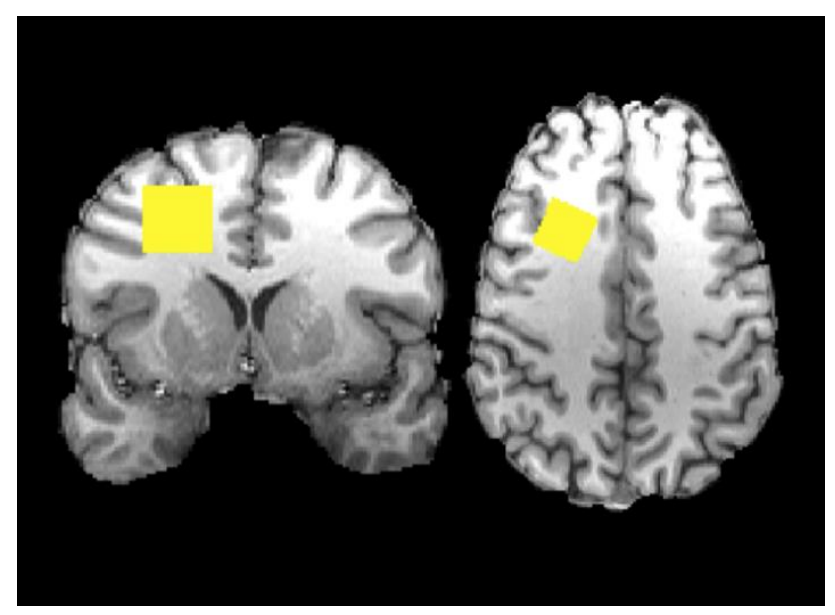

Figure 2

Voxel spectra for each participant.

The left column shows the whole spectrum with the LC model fit (red), the residual (top black line), GABA (blue line) and glutamate (green line). The $x$-axis shows the chemical shift in parts per million (ppm) and ranges from 4.2 to $0.6 \mathrm{ppm}$. 


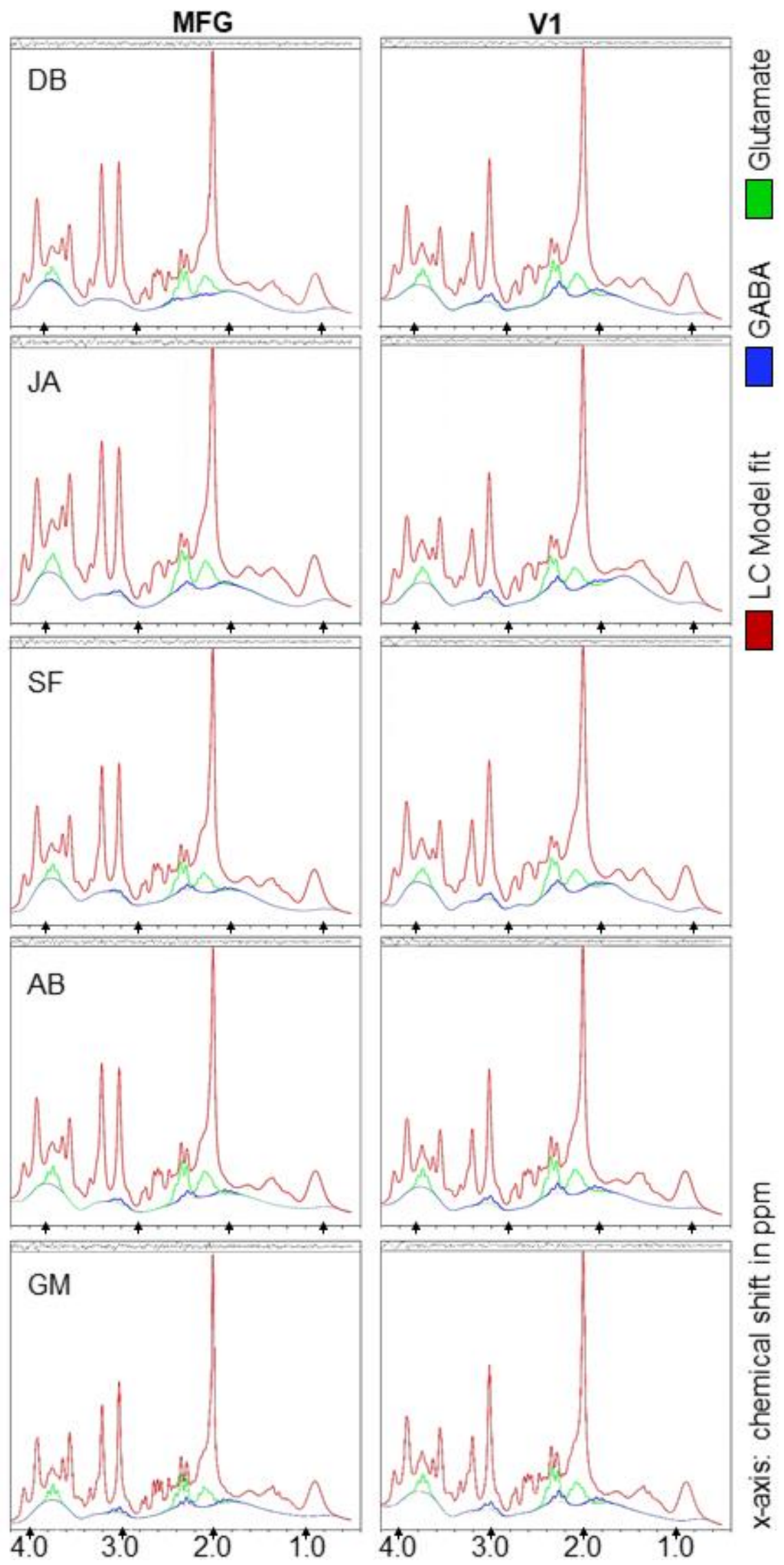




\section{Figure 3}

\section{Reduced glutamate/GABA in a calculation prodigy.}

Glutamate/GABA concentrations per participant (means and standard deviations for the control group and mean for G.M.). A) G.M.'s MFG ratio was $22.8 \%$ lower than the group average ( $p=.04$, two-tailed). B) There was no difference in V1.
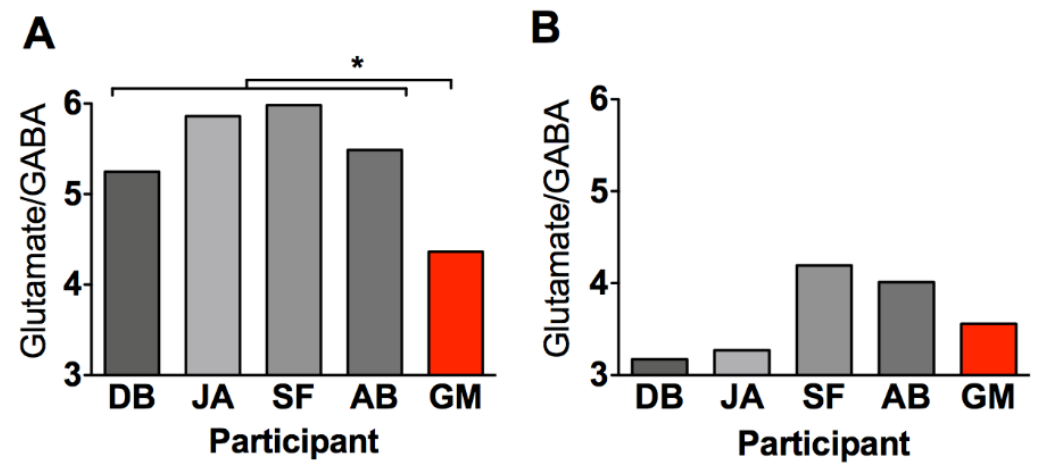


\begin{tabular}{|c|c|c|c|c|c|}
\hline Participant & DB & JA & SF & $A B$ & G.M. \\
\hline Age & 45 & 49 & 42 & 41 & 46 \\
\hline $\begin{array}{l}\text { Years of } \\
\text { higher } \\
\text { education }\end{array}$ & 11 & 9 & 13 & 6 & 11 \\
\hline $\begin{array}{l}\text { Mathematics } \\
\text { composite } \\
\text { (standard } \\
\text { score) }\end{array}$ & 126 & 128 & 120 & 116 & 143 \\
\hline $\begin{array}{l}\text { Mathematics } \\
\text { percentile }\end{array}$ & 96 & 97 & 91 & 86 & 99.8 \\
\hline Study topic & $\begin{array}{l}\text { Combustion } \\
\text { engineering } \\
\text { and artificial } \\
\text { intelligence }\end{array}$ & $\begin{array}{l}\text { Mathematical } \\
\text { sciences and } \\
\text { physics }\end{array}$ & $\begin{array}{l}\text { Psychology, } \\
\text { physics, } \\
\text { medical } \\
\text { physics }\end{array}$ & Physics & $\begin{array}{l}\text { Mathematics, } \\
\text { psychology } \\
\text { and } \\
\text { education }\end{array}$ \\
\hline
\end{tabular}

\section{Table 1}

\section{Standardized calculation abilities.}

G.M. and male control participants matched for age, education and standardized mathematical scores. All participants worked in psychology and mathematics-related science. 


\begin{tabular}{|c|c|c|c|c|c|c|}
\hline Participant & $D B$ & $J A$ & $S F$ & $A B$ & G.M. & $\begin{array}{l}\text { t- } \\
\text { statistic, } \\
\text { p-value } \\
\text { (Cohen's } \\
\text { d) }\end{array}$ \\
\hline \multicolumn{7}{|c|}{ MFG } \\
\hline $\begin{array}{l}\text { GABA } \\
(C R L B \%)\end{array}$ & $\begin{array}{l}.044 \\
(17)\end{array}$ & $\begin{array}{l}.032 \\
(20)\end{array}$ & $\begin{array}{l}.025 \\
(19)\end{array}$ & $\begin{array}{l}.028 \\
(14)\end{array}$ & $\begin{array}{l}.041 \\
(14)\end{array}$ & $\begin{array}{l}T=.94 \\
p=.42 \\
(d=1.09)\end{array}$ \\
\hline $\begin{array}{l}\text { Glutamate (CRLB } \\
\%)\end{array}$ & $.23(3)$ & $.19(3)$ & $.15(3)$ & $.16(3)$ & $.18(4)$ & $\begin{array}{l}\mathrm{T}=-.07 \\
p=.95 \\
(\mathrm{~d}=.08)\end{array}$ \\
\hline GIu/GABA & 5.25 & 5.86 & 5.98 & 5.49 & 4.36 & $\begin{array}{l}\mathrm{T}=-3.39, \\
p=.04 \\
(d=3.95)\end{array}$ \\
\hline \multirow[t]{2}{*}{$\begin{array}{l}\text { Linewidth } \quad(\mathrm{Hz}) \\
(S N R)\end{array}$} & $\begin{array}{l}4.43 \\
(77)\end{array}$ & $\begin{array}{l}5.41 \\
(67)\end{array}$ & $\begin{array}{l}3.94 \\
(80)\end{array}$ & $\begin{array}{l}3.94 \\
(84)\end{array}$ & $\begin{array}{l}2.95 \\
(69)\end{array}$ & $\begin{array}{l}p=.15 \\
(\mathrm{SNR}: \\
p=.4)\end{array}$ \\
\hline & $27 / 70 / 3$ & $21 / 75 / 4$ & $18 / 79 / 3$ & $15 / 83 / 2$ & $19 / 79 / 2$ & $\begin{array}{l}\mathrm{GM}: \mathrm{T}=- \\
.22, \\
p=.84, \\
(\mathrm{~d}=.97)\end{array}$ \\
\hline$(G M+W M) /(G M+W M+C S F)$ & .97 & .96 & .97 & .98 & .98 & $\begin{array}{l}\mathrm{T}=.73 \\
p=.52\end{array}$ \\
\hline $\begin{array}{l}\text { Averages } \\
\text { removed/analyzed }\end{array}$ & $2 / 126$ & $16 / 112$ & $18 / 119$ & 2/126 & $8 / 120$ & $\begin{array}{l}T=-.1, \\
p=.93(d=- \\
.12)\end{array}$ \\
\hline
\end{tabular}




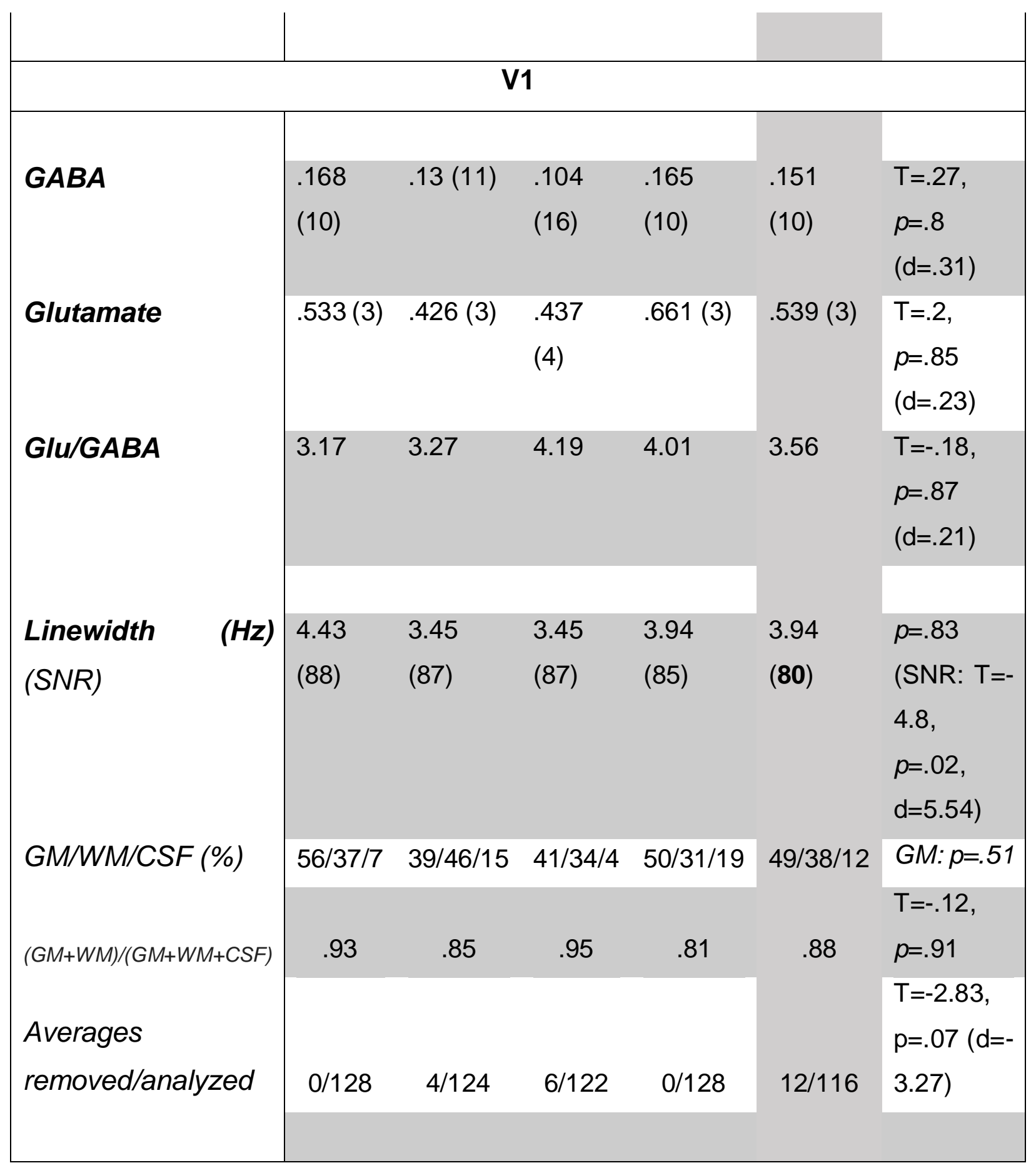

\section{Table 2}

Regional neurotransmitter concentrations for the calculation prodigy and controls. Glutamate/GABA ratios are significantly lower in G.M. compared to controls. For GABA alone, there was only a trend. Cramer-Rao lower bounds (CRLBs) below $20 \%$ indicate good reliability of the acquired concentrations per region. Line widths (full-width-halfmaximum, FWHM) are shown in $\mathrm{Hz}$, and in brackets signal-to-noise ratio (SNR). In V1, 
the SNR was significantly lower in G.M. compared to controls ( $p=.02$ ). Bold print highlights where G.M.'s value differs significantly from the controls'. Voxel tissue content shown in percent (gray matter/white matter/CSF), as well as the number of bad averages removed vs. included in the analysis. 


\begin{tabular}{|c|c|c|c|c|c|c|}
\hline Participant & DB & JA & SF & AB & G.M. & $\begin{array}{l}\text { t- } \\
\text { statistic } \\
(\mathrm{df}=3), \\
p \text {-value } \\
\text { (Cohen's } \\
\text { d) }\end{array}$ \\
\hline \multicolumn{7}{|c|}{ Right caudal middle frontal gyrus (Freesurfer ROI) } \\
\hline $\begin{array}{l}\text { Gray matter } \\
\text { volume }\end{array}$ & 5869 & 4236 & 6499 & 6451 & 7434 & $\begin{array}{c}T=1.41, \\
p=.25 \\
(d=1.63)\end{array}$ \\
\hline Cortical thickness & 2.434 & 2.279 & 2.403 & 2.609 & 2.445 & $\begin{array}{l}T=.09 \\
p=.93 \\
(d=.1)\end{array}$ \\
\hline Mean curvature & .125 & .113 & .127 & .118 & .123 & $\begin{array}{c}T=.31 \\
p=.78 \\
(d=.36)\end{array}$ \\
\hline Surface area & 2235 & 1690 & 2415 & 2298 & 2763 & $\begin{array}{c}T=1.68, \\
p=.19 \\
(d=1.94)\end{array}$ \\
\hline $\begin{array}{l}\text { Total cortical } \\
\text { volume }\end{array}$ & 485947 & 457607 & 502459 & 423108 & 524082 & $\begin{array}{c}\mathrm{T}=1.46, \\
p=.24 \\
(\mathrm{~d}=1.69)\end{array}$ \\
\hline $\begin{array}{l}\text { Intracranial } \\
\text { volume }\end{array}$ & 1418843 & 1488273 & 1513460 & 1166900 & 1468258 & $\begin{array}{c}T=.21, \\
p=.85 \\
(d=.24)\end{array}$ \\
\hline
\end{tabular}

Table 3. 
Gray matter region of interest $(\mathrm{ROI})$ analysis. One-tailed Crawford's t-tests showed no differences in the Freesurfer parcellated ROI (right caudal middle frontal gyrus, DesikanKillany atlas). There were no gray matter differences in the right caudal MFG or the whole brain compared to controls. 